\title{
Improved Interfaces for Human-Robot Interaction in Urban Search and Rescue*
}

\author{
Michael Baker, Robert Casey, Brenden Keyes, and Holly A. Yanco \\ Computer Science Department \\ University of Massachusetts Lowell \\ One University Avenue, Olsen Hall \\ Lowell, MA 01854 USA \\ \{mbaker, rcasey, bkeyes, holly\}@cs.uml.edu
}

\begin{abstract}
Studies of human-robot interaction have shown that operators rely heavily upon the video stream, to the exclusion of all other information on the interface. We have created a new interface that fuses information on and around the video window to exploit this fact.
\end{abstract}

Keywords: Human-robot interaction, search and rescue.

\section{Introduction}

Robots can assist human teams in urban search and rescue (USAR) tasks by traveling into dangerous and small areas to search for survivors. Robots need to climb on and maneuver around rubble piles that are deemed too dangerous or hazardous for human or animal searchers to investigate. In difficult USAR environments, it is not yet possible to create a fully autonomous robot to completely take the place of a human rescue worker. In fact, most USAR robots that are sent into disaster zones are teleoperated. For this application, operators must have a good awareness of their surroundings, yet it is difficult to obtain situation awareness [1,2].

We have performed studies on more than a dozen USAR interfaces used in the American Association for Artificial Intelligence (AAAI) and RoboCup Robot Rescue competitions and have also done usability testing with domain experts at the National Institute of Standards and Technology (see, for example, [1], [2] and [3]). These studies allowed us to identify the successes and failures of different interfaces, and we have developed guidelines for the effective design of interfaces for human-robot interaction in a USAR application [4]:

Enhance awareness. Provide a map indicating where the robot has been. Provide more spatial information about the robot in the environment to make operators more aware of their robots' immediate surroundings.

Lower cognitive load. Provide fused sensor information rather than make the user mentally combine data from multiple sources.
Increase efficiency. Minimize the use of multiple windows, and provide user interfaces that support multiple robots in a single window, if possible.

Provide help in choosing robot modality. Provide the operator assistance in determining the most appropriate level of robotic autonomy at any given time.

We have observed that most users focus on one area of the interface during most of the testing: the area that displays the robot's video feed. Additionally, we have observed that large portions of most USAR graphical user interfaces (GUI) are "dead space." By dead space, we mean aspects of the GUI that either do not work or have little relevance to the task or individual user. During studies, users have asked questions about or expressed frustration at useful, but broken, functionality on a GUI. Broken or unused areas of a GUI take away valuable real estate from the display of useful information.

\section{Designing a New Interface}

To test the design guidelines above and to determine how to make the best use of available screen real estate, we are modifying a robot system designed by INEEL. The INEEL system $[5,6]$, under development for over three years, incorporates a well-tested user interface as well as a robot with multiple autonomy modes.

INEEL's navigation system consists of four autonomy modes: teleoperation, safe, shared, and autonomous. In the teleoperation mode, the robot's operator makes all of the decisions regarding the robot's movement. In safe mode, the operator is still directing the robot, but the robot will not allow the operator to drive it into obstacles. In shared mode, the user can indicate which direction to travel in and the robot will safely drive in the specified direction. The autonomous mode can be used as a wander mode; it can also return to a prior location on the robot-generated map.

INEEL's interface, shown in figure 1, contains a wealth of information. In the upper left corner, a window contains the video stream from the robot. Buttons for

\footnotetext{
${ }^{*}$ 0-7803-8566-7/04/\$20.00 @ 2004 IEEE.
} 


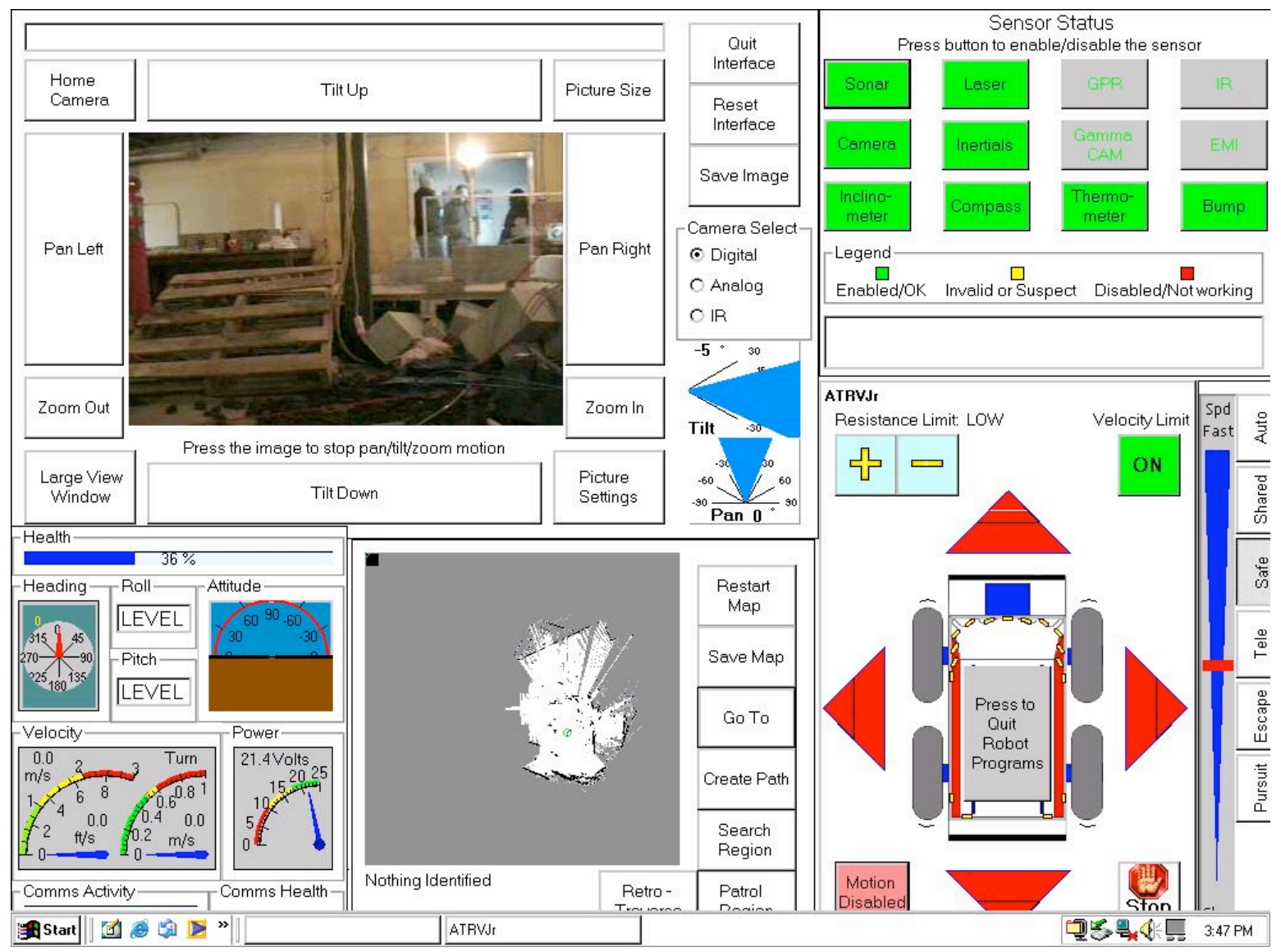

Figure 1: The INEEL interface for their robotic system.

panning, tilting and zooming the camera are around the video window. Moving to the right, there are pan and tilt indicators and a camera selection area. On the right corner of the interface are status indicators for the various sensor systems on the robot. The indicators will be green if the sensors are working properly, yellow if the sensor readings are suspect, and red if the sensors have been disabled (the operator can turn off sensor systems by selecting these buttons). Below this area is a sensor map, where the readings of the distance sensors are shown. The four sets of triangles on each side of the robot will fill with more and more red as objects approach. The triangles are also used to show the direction of movement, filling with some green to indicate direction and speed. To the right of the sensor map is a speed control slider and buttons to select the desired autonomy mode. To the left of the sensor map is the map of the environment created by the robot as it moves around. To the left of the environment map is an area that gives additional status information about the robot, including battery level and tilt.

While an experienced user might be able to use all of the information on the interface screen, we found in our tests that most users focused exclusively on the video window during their runs. When teleoperating a robot, it is very important to have a good video stream because the operator needs to watch the video closely to drive effectively. The operator also needs to use the video to locate victims in the environment. In a study of how operators acquire situation awareness, we found that an average of $30 \%$ of run time was spent looking around the environment to the exclusion of all other tasks (i.e., just looking, not navigating at the same time) [2].

To lower the cognitive load on the user, our interface combines information that previously existed in multiple areas of the screen. Our interface capitalizes on the user's natural focus on the video window by bringing more information to this area. Some examples of relocated information are described in the subsections below.

\subsection{Pan and Tilt Indicators}

We have observed in multiple studies that operators often forget to recenter the camera after panning and tilting the camera. Failing to recenter the camera before starting to drive the robot can result in a loss of situation awareness, since the camera is pointing a different way than the operator would expect. For example, in one study [3], we found that an operator drove with his camera off center for over half of his run, causing him to hit more obstacles. The camera pointed left and the operator saw a clear area, but there was an obstacle in front of the robot. 


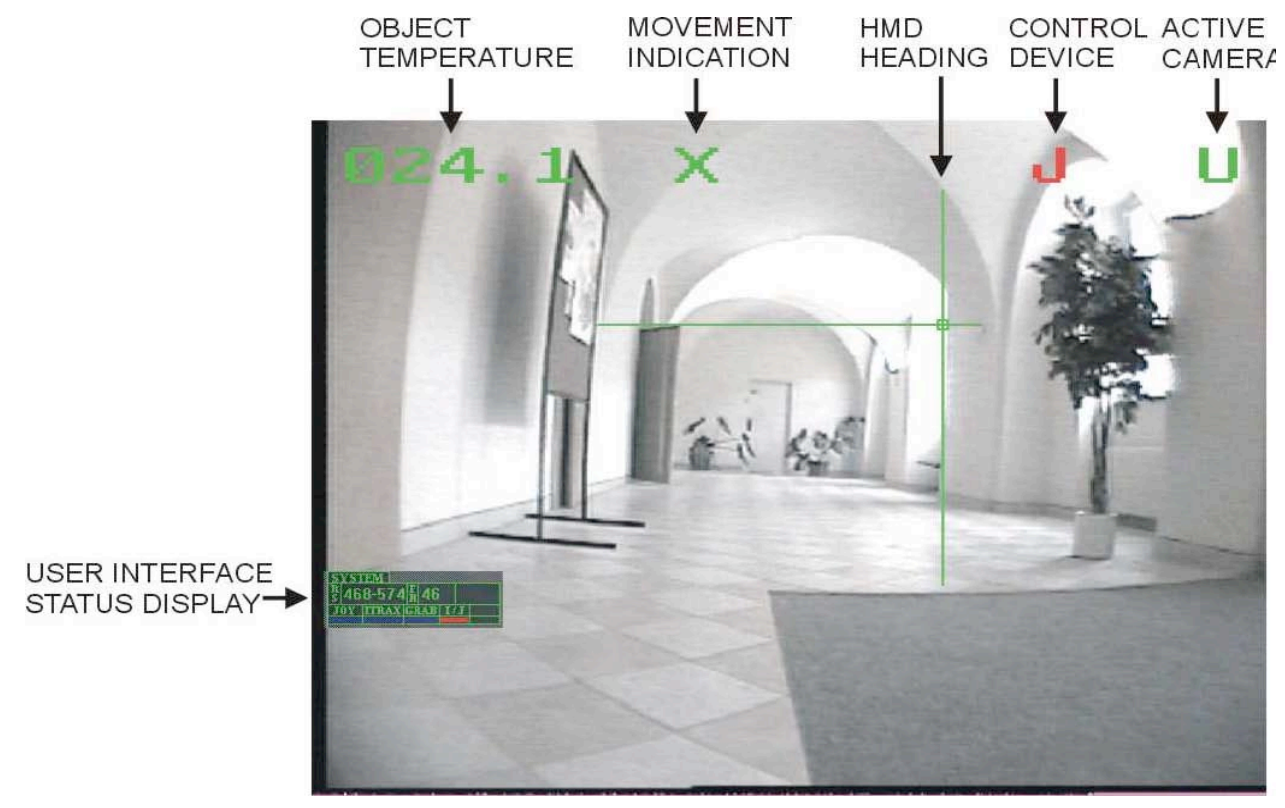

Figure 2: RoBrno's interface, showing crosshairs to indicate the camera position (from [7]).

When switching from looking around the environment to driving the robot, operators often forget to change the camera view. One option to correct this problem would be to automatically center the camera when the operator starts driving. However, there might be times where an operator would like to look along a wall to the left while moving forward. In this case, we would like to allow the camera to remain pointing to the left.

Instead of making an automatic adjustment, we choose instead to make a more visible reminder of the camera's attitude. The INEEL interface provides pan and tilt indicators, but operators may not notice them immediately. Rather than having separate indicators for the pan and tilt of the robot's camera, our interface overlays a light cross on the screen to indicate the direction in which the camera is pointing. These crosshairs were inspired by RoBrno, a robot system that uses a heads-up display [7]. (RoBrno's interface is shown in figure 2.)

We capitalize on the presence of the crosshairs by also using them to display distance of objects from the robot. To do this, we place hash marks on the vertical line of the cross at 1/2-meter spacings.

\subsection{Ranging Information}

Instead of displaying ranging information gathered from sonars and laser ranging in a separate window, we place these values in colored blocks around the video window. The colored blocks range from red (very close obstacle) to yellow (obstacle approaching) to green (clear in that direction). The user can choose to have numeric distances displayed in the boxes as well.
Moving the ranging information so that it surrounds the video window should allow the operator to immediately see that the sonar and ladar sensors are detecting obstacles. When there are no obstacles, the boxes blend into the background. As obstacles get closer, the boxes change to brighter colors, changing the area just around the operator's focus on the video window.

\subsection{System Alerts}

Rarely consulted information on the interface is now treated as a system alert. For example, we have not observed users looking at the battery level during usability tests. We have kept a small meter on the screen, but, more importantly, bring up an alert when the user has used almost half of the robot's power during the run, since the robot will need the remaining power to get back to the starting area.

We are also sending system alerts when sensor readings are suspect instead of using the multiple button area from the INEEL interface's upper right hand corner.

\subsection{Autonomy Suggestions}

The system has four autonomy modes: teleoperation (no sensors are used to help keep the robot from bumping into objects), safe (teleoperation with obstacle avoidance provided by the system), shared (semi-autonomous navigation with obstacle avoidance where the user communicates his desires at points in the route where a choice must be made or can otherwise bias the robot's travel direction), and autonomous (the robot is given a goal point to which it then safely navigates). To help the user choose the correct mode, we are investigating ways to 

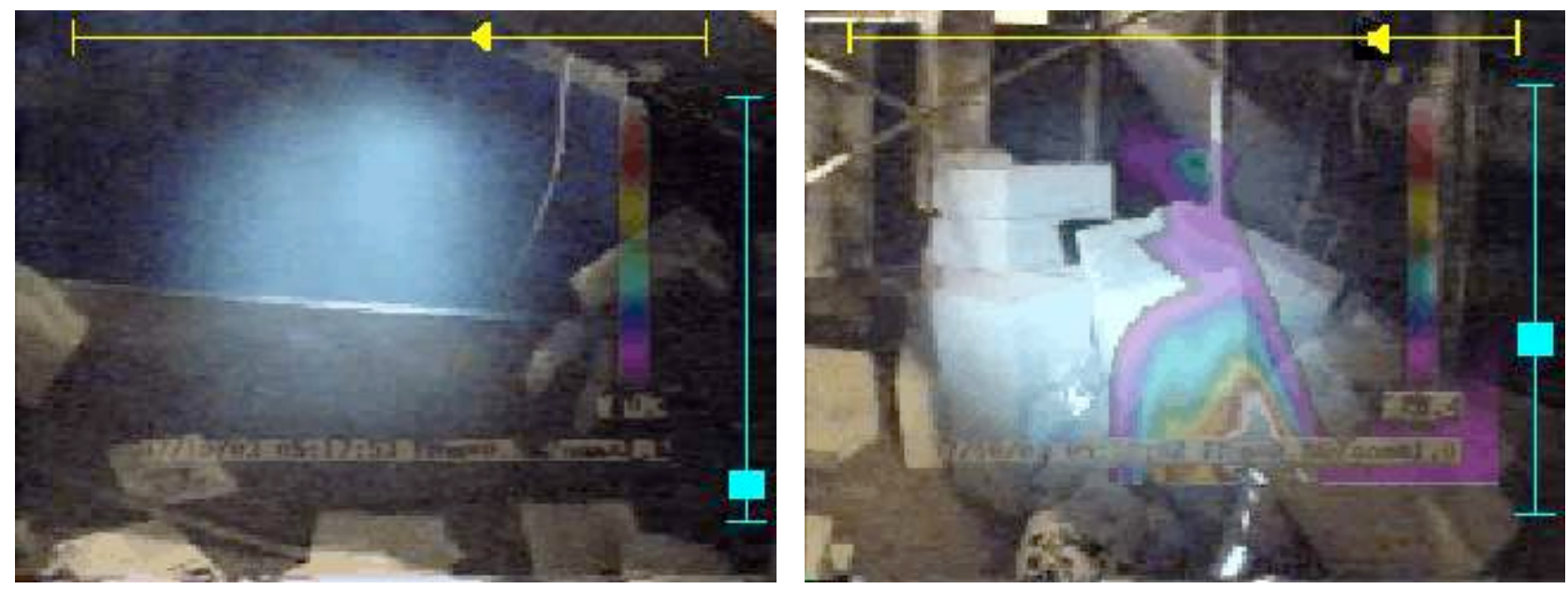

Figure 3 : Information fusion, from [9]. The figures above show a combination of color video, thermal imaging, direction of loudest sound and a carbon dioxide reading. This fused display will be integrated into our interface.

suggest autonomy mode changes [8]. The system's suggestions appear in a bar just below the video window, keeping the user's attention on that primarily used area.

\subsection{Map of the Environment}

By consolidating information around and in the video window, we have freed up space on the interface screen, which will allow us to move the robot-generated map of the environment to the same eye level as the video screen. We will also include additional information on the map. The original INEEL system showed a light dot in the robot's location, but most of the people using the system did not see it. We are emphasizing the placement of the robot on the map and also giving an indication of the direction in which the robot is pointing. These changes should enhance the user's awareness of the robot's position in the environment.

\subsection{Customization}

We have observed a low level of customization in the interfaces that we have studied. In fact, most interfaces allow for no customization at all. Often, a USAR interface reflects a developer's preference or convenience. The interfaces are designed to support research, and not necessarily with the intended end user as a primary consideration. Hence, USAR interfaces are not simple to learn, to understand, or to use.

HRI studies have shown that different people interact differently with a particular interface. Moreover, the differences seem to be independent of age, gender, and experience with robotic teleoperation [6]; i.e., different people work differently. We are investigating interface customizations that will allow users to be as efficient as possible. Our design also allows the user to hide useless (from the individual user's perspective) aspects of the interface.

\subsection{Adding Sensors to the Rear of the Robot}

In usability experiments [2], we observed the robot bump obstacles in the environment an average of 2.6 times per run. Of the 29 hits during all of the four subjects' runs, 12 or $41 \%$ of the hits were on the rear of the robot. We believe a lack of sensing is causing many of the rear hits.

To address the issue of poor situation awareness in the back of the robot, we have added a rear-looking camera to our system. Since the rear-looking camera will only be consulted occasionally, and we do not wish to draw attention away from the main video feed, the rear video feed is relegated to a smaller window and updated less frequently. We have placed it above the main video window in a similar fashion to a rear view mirror on a car.

We can switch the video displays so that the rear view is expanded in the larger window. The large display indicates whether the front or rear view is active. Also, the drive commands automatically remap so that forward becomes reverse and reverse becomes forward. The command remapping allows an operator to spontaneously reverse the direction of the robot in place and greatly simplifies the navigation task.

\subsection{Additional Sensor Fusion}

We are also investigating how we could provide additional sensor fusion on the interface. For this work, 


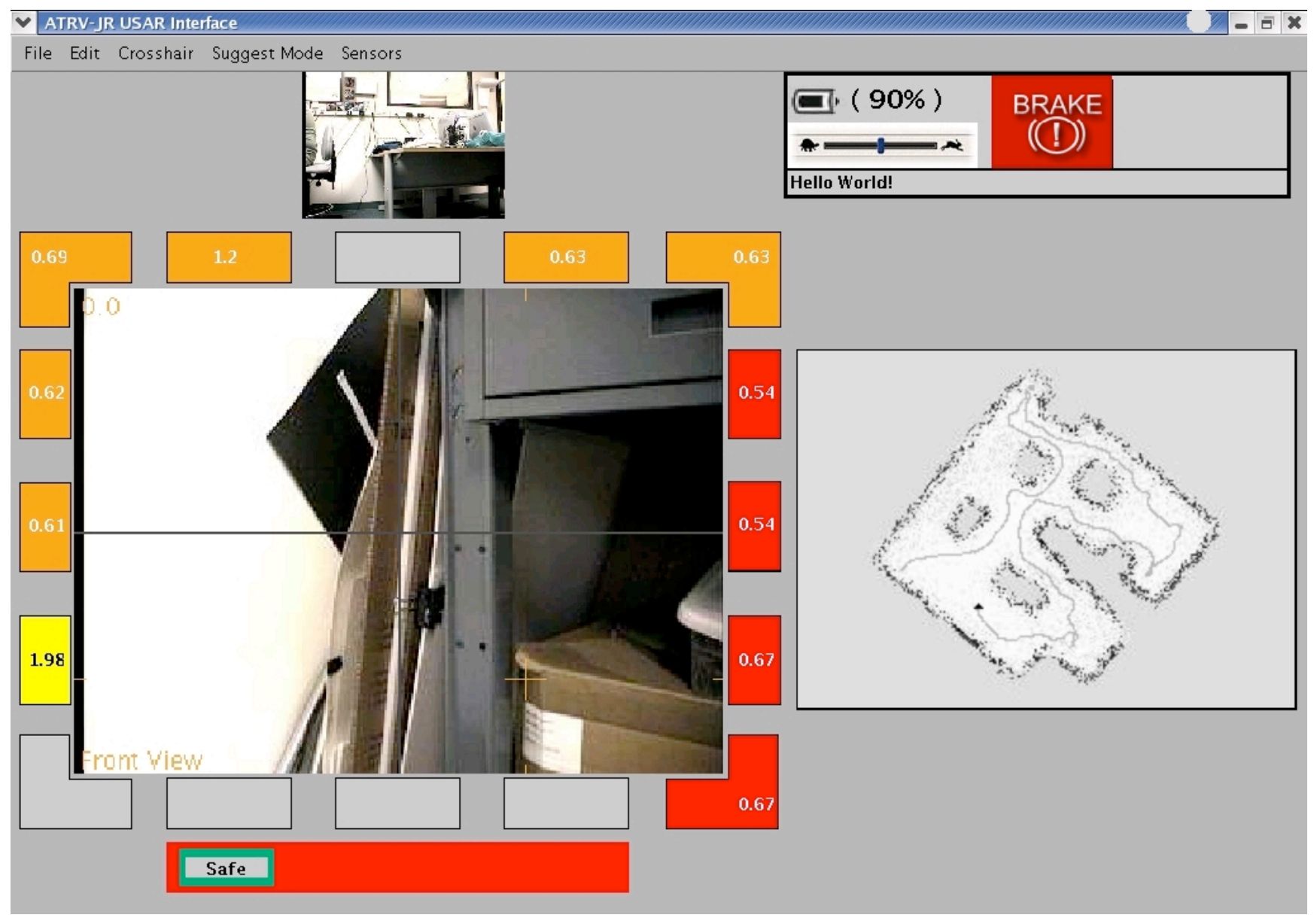

Figure 4: Our interface design. On the left is the video window, surrounded by the readings of the distance sensors on the robot. Crosshairs indicate the position of the camera. The upper video window shows a rear camera view. The robotgenerated map will be placed to the right of the video window in the interface (the map shown is for illustration).

we will integrate a display that fuses color video, forwardlooking infrared (heat detection), sound, and carbon dioxide [9]. Figure 3 shows two examples of this fused display.

\section{Discussion and Conclusions}

Our new interface is shown in Figure 4. The left of the screen shows the video window surrounded by the ranging information. Crosshairs overlayed on the video show the camera's attitude. Above this video window is a rear view video. Under the video window is the mode suggestion area. To the right of the video window is the robot-generated map. Finally, above the map is the alerts area.

Five subjects have used our interface, all of whom found the interface easy to use. We have not yet had the opportunity to directly compare our new interface to INEEL's interface. We expect that most operators will find the new interface easier to use, but that more experienced operators may miss some of the additional status information that we have removed.

\section{Acknowledgments}

This work is supported in part by NSF IIS-0308186 and NIST 70NANB3H1116. Rachel Mulcrone assisted with the evaluation of the interface design.

\section{References}

[1] Drury, J., J. Scholtz and H.A. Yanco (2003). "Awareness in human-robot interactions." Proceedings of the 2003 IEEE Conference on Systems, Man and Cybernetics.

[2] Yanco, H.A. and J.L. Drury (2004). “"Where am I?' Acquiring situation awareness using a remote robot platform." Proc. 2004 IEEE Conference on Systems, Man and Cybernetics, this volume. 
[3] Yanco, H.A., J.L. Drury and J. Scholtz (2004). "Beyond usability evaluation: analysis of human-robot interaction at a major robotics competition." HumanComputer Interaction, Vol. 19, No. 1 \& 2, pp. 117 149.

[4] Drury, J.L., J. Scholtz, and H.A. Yanco "Applying CSCW and HCI techniques to human-robot interaction." Proc. CHI 2004 Workshop on Shaping Human-Robot Interaction, April 2004, Vienna, pp. 13-16.

[5] Bruemmer, D.J., D.D. Dudenhoeffer, and J. Marble (2002). "Dynamic Autonomy for Urban Search and Rescue." AAAI Mobile Robot Workshop, Edmonton, Canada, August 2002.

[6] Bruemmer, D.J., R.L. Boring, D.A. Few, and J.L. Marble (In submission). "Novice users of a robotic interface in a search and rescue task." Submitted to CHI2004.

[7] Zalud, Ludek (2003). "RoboCup Rescue Robot League Competition Awardee Paper: RoBrno, Czech Republic, $1^{\text {st }}$ Place." Padova, Italy, July. Downloaded from

http://www.isd.mel.nist.gov/projects/USAR/Robrno\%20 Awardee\%20Paper.pdf on 30 June 2004.

[8] Baker, M. and H.A. Yanco (2004). “Autonomy mode suggestions for improving human-robot interaction." Proc. 2004 IEEE Conference on Systems, Man, and Cybernetics, this volume.

[9] Hestand, D. and H.A. Yanco (2004). "Layered sensor modalities for improved human-robot interaction." Proc. 2004 IEEE Conference on Systems, Man, and Cybernetics, this volume. 